Cahiers $d u$ MONDE RUSSE

\section{Cahiers du monde russe}

Russie - Empire russe - Union soviétique et États indépendants

55/3-4 | 2014

Varia

\title{
Boris Petric, éd., La Fabrique de la démocratie, ONG, Fondations, think tanks et organisations internationales en action
}

\section{Françoise Daucé}

\section{(2) OpenEdition}

1 Journals

\section{Édition électronique}

URL : http://journals.openedition.org/monderusse/8153

DOI : 10.4000/monderusse.8153

ISSN : $1777-5388$

Éditeur

Éditions de l'EHESS

\section{Édition imprimée}

Date de publication : 1 juillet 2014

Pagination : 527-530

ISBN : 978-2-7132-2441-6

ISSN : $1252-6576$

\section{Référence électronique}

Françoise Daucé, «Boris Petric, éd., La Fabrique de la démocratie, ONG, Fondations, think tanks et organisations internationales en action », Cahiers du monde russe [En ligne], 55/3-4 | 2014, mis en ligne le 14 avril 2015, Consulté le 24 septembre 2020. URL : http://journals.openedition.org/monderusse/ 8153 ; DOl : https://doi.org/10.4000/monderusse.8153

Ce document a été généré automatiquement le 24 septembre 2020.

(c) École des hautes études en sciences sociales 


\title{
Boris Petric, éd., La Fabrique de la démocratie, ONG, Fondations, think tanks et organisations internationales en action
}

\author{
Françoise Daucé
}

\section{RÉFÉRENCE}

Boris PETRIC, éd., La Fabrique de la démocratie, ONG, Fondations, think tanks et organisations internationales en action, Paris : Éditions de la Maison des Sciences de l'Homme, 2012, $310 \mathrm{p}$.

1 Cet ouvrage collectif, fruit d'un projet de recherche réunissant des anthropologues, des sociologues et des politistes, analyse l'action de démocratisation des agences de coopération, des $\mathrm{ONG}$, des fondations et des organisations internationales dans des États sortant de l'autoritarisme ainsi que leur réception par les acteurs locaux. Le livre regroupe onze contributions, portant sur des études de cas extrêmement diverses, allant du Sénégal (étudié par G. Blundo) à Cuba (D. Dimitrijevic) en passant par le Kirghizstan (B. Petric), la Bulgarie (D. Anguelova-Lavergne), l'Azerbaïdjan (R. Mathey), la Bolivie (D. Recondo), l'Afghanistan (A. Monsutti), la Serbie (T.Vetta), le Pakistan (S. Hussain), l'Indonésie (R. Bertrand) et le Nigeria (M.-A. Pérouse de Montclos). Renonçant à l'analyse des discours sur la démocratie, la réflexion porte sur les effets sociaux et politiques de la généralisation de l'assistance à la bonne gouvernance depuis la disparition du bloc socialiste. Les auteurs insistent sur la coproduction des pratiques de gouvernement par les acteurs internationaux, les États et les acteurs locaux. Ils s'interrogent sur la logique des programmes de développement, sur leurs effets sur la gouvernementalité néolibérale (autour d'une référence commune aux travaux de J. Ferguson et A. Gupta ${ }^{1}$ ) et sur les nouvelles formes de domination sur les États et les sociétés qu'ils produisent. Face à la diversité des cas présentés, la lecture peut 
s'articuler autour de trois questions centrales qui trouvent des échos dans les diverses contributions : les effets de l'intervention internationale sur la professionnalisation des acteurs locaux du développement, la corrélation entre cette professionnalisation et le retrait de ces acteurs du champ politique électoral et, enfin, les formes imprévues de domination engendrées par ces effets de professionnalisation et de dépolitisation.

2 Dans une approche commune, inspirée notamment par la lecture des travaux de Nicolas Guilhot ${ }^{2}$, les contributeurs accordent une attention particulière aux professionnels des programmes de démocratisation (les « courtiers en développement ») qui interviennent dans les États dits «en transition démocratique ». Ils soulignent que leurs actions ont pour premier effet, direct et mesurable, la professionnalisation des militants du développement local dans les différents contextes étudiés. Les acteurs autochtones apprennent ainsi à traduire leur engagement dans le langage des grants (subventions internationales), de l'assistance technique et de l'action par projets. Au Kirghizstan, la création des ONG du haut vers le bas renforce leur caractère artificiel, car les salariés de ces organisations sont financés par l'international. En Serbie, les ONG locales essayent de devenir des prestataires de services, des organisateurs, des consultants ou des évaluateurs des réformes. Elles sont aidées par les programmes de "développement de la société civile " qui organisent des formations à la planification stratégique, à l'apprentissage de la prise de parole en public (voire à la méditation new-age !). Cette professionnalisation ne préjuge cependant pas des résultats de leur action en termes de développement démocratique. Au Nigeria, les fondations politiques apprécient généralement leur impact à l'aune du nombre de séminaires réalisés, de réunions organisées, d'ouvrages publiés sans mesurer l'influence de ces actions sur le public. Ce constat conduit Marc-Antoine Pérouse de Montclos à se demander quel est l'objectif réel des « courtiers de la démocratisation » dans ce pays.

3 Les auteurs soulignent à plusieurs reprises les difficultés posées par le passage des promoteurs du développement démocratique à l'engagement politique (compris ici comme un engagement dans les partis politiques et dans le jeu électoral). Ils reprennent la thèse de J. Ferguson qui souligne que les rouages du développement constituent une "machine antipolitique $»^{3}$. Les agents de la démocratisation s'appuient sur leur expertise pour participer à la transformation des espaces politiques locaux tout en refusant généralement de s'adonner à la compétition électorale. C'est bien le cas au Sénégal où la professionnalisation du Forum civil, étudié par Giorgio Blundo, ne se traduit pas nécessairement par une participation des militants à la vie politique électorale mais par leur influence indirecte sur l'État par l'intermédiaire de rapports sur la corruption. En Bulgarie, les ONG et think tanks engagés dans les réformes libérales ne participent pas aux processus politiques. Ils mènent leur action en dehors du cadre de la représentativité électorale, ce qui aboutit à une forme de négation des mécanismes institutionnels de la démocratie représentative. En Afghanistan, les experts humanitaires et du développement ont tendance à négliger la relation politique. La question de leur légitimité et de la représentation populaire est passée sous silence. Même lorsque les élites circulent entre les institutions étatiques et non étatiques, comme en Serbie, les responsables associatifs offrent leur expertise et considèrent que " la politique est un sale boulot ». Après la chute de Milosevic en 2000, l'activisme politique a cédé la place au professionnalisme et à la bureaucratie. Ce constat partagé pose la question du passage d'une vision tocquevillienne à une vision gramscienne de la société civile et des ONG. Dans la vision de Gramsci, les ONG peuvent 
porter une forme de résistance sociale apte à remettre en cause des relations capitalistes de domination. Dans les cas présentés ici, les programmes de développement favorisent plutôt l'instauration de nouvelles formes de contraintes publiques appuyées sur l'expertise, aux dépens de la compétition et de la critique politiques.

4 Les auteurs soulignent ainsi que les programmes de démocratisation portent de nouvelles formes de domination. En Bolivie, l'ajustement structurel et la thérapie de choc imposés par Washington ont été appropriés de façon originale par les acteurs locaux. La mise en œuvre de la décentralisation ne répond ni tout à fait aux attentes des acteurs internationaux, ni tout à fait à celle des acteurs locaux. Dans le cas bolivien, on assiste à une communautarisation imprévue de la participation politique locale alors que la réforme de l'administration territoriale était d'inspiration libérale. En Indonésie, Romain Bertrand examine comment les programmes internationaux d'aide à la démocratisation entrent en résonance avec une tradition de pensée locale hostile au multipartisme concurrentiel et favorable à une restriction forte du nombre de compétiteurs partisans. Ainsi, après la disparition du régime autoritaire du général Suharto, le choix indonésien du pluripartisme limité et de la judiciarisation accrue du jeu électoral est imputable au pouvoir d'influence des "ingénieurs de la démocratie " étrangers mais aussi au "nationalisme aristocratique» indigène. À Cuba, la libéralisation de l'économie cubaine ne s'accompagne pas d'une volonté de démocratisation politique, les individus impliqués dans la nouvelle économie libéralisée étant même les plus nostalgiques de la période soviétique. Dans les cas les plus extrêmes, les usages imprévus des programmes de démocratisation aboutissent à un renouvellement des formes de l'autoritarisme. Cet ouvrage sur la fabrique de la démocratie pose ainsi indirectement la question de la fabrique des nouveaux régimes autoritaires appuyés sur les pratiques de démocratisation. Au Kirghizstan, la captation de l'assistance à la démocratie par Bakiev, président du pays de 2005 à 2010, contribue à la construction de son autoritarisme et pose la question de la responsabilité des organisations internationales dans cette dérive. Au Nigeria, M.-A. Pérouse de Montclos considère que les fondations allemandes ont eu historiquement pour objectif de susciter des alliances stratégiques contre la menace communiste, n'hésitant pas à collaborer avec des régimes autoritaires, et qu'elles restent avant tout des relais des réseaux diplomatiques de Berlin.

5 L'effort de problématisation commune des auteurs donne à cet ouvrage, dont la diversité fait la richesse, sa qualité. Fondé sur des enquêtes de terrain et des données empiriques d'une grande précision, il documente concrètement l'effet des politiques de démocratisation, généralement conçues comme l'application de quelques recettes universelles, dans des contextes politiques et sociaux très divers et leurs conséquences sur la transformation des espaces politiques concernés. L'un des paradoxes de cet ouvrage, s'il faut en relever un, réside peut-être dans le décalage entre la volonté affichée (en introduction) d'examiner les formes de transnationalisation du politique et la construction du livre autour de chapitres traitant de cas nationaux. Ce cadrage conduit presque mécaniquement les auteurs à souligner que la « référence à l'État n'a jamais été aussi importante qu'en ces temps marqués, pourtant, par la vulgate néolibérale ». Si le cadre étatique permet de donner des éclairages intéressants sur les effets politiques imprévus des programmes de démocratisation internationaux sur différents régimes et dans différentes sociétés, il ne facilite pas la lecture transversale des évolutions observées dans ces divers contextes. Pour ce faire, une enquête mettant 
l'accent sur les connexions et les convergences en cours d'un espace à l'autre permettrait de compléter l'analyse. En se détachant du cadre national pour faire la sociologie de l'action transnationale à des échelles qui peuvent être inférieures ou supérieures à l'État, de nouvelles perspectives pourraient s'ouvrir dans le prolongement de cet ouvrage déjà fort riche.

\section{NOTES}

1. J. Ferguson, A.Gupta, "Spatializing states: toward an ethnography of neoliberal governmentality », American Ethnologist, 29 (4), p. 981-1002.

2. N. Guilhot, The Democracy Makers: Human Rights and the Practices of Global Order, New-York: Columbia University Press, 2005.

3. J. Ferguson, The Anti-Politics Machine: Development, Depoliticization and Bureaucratic Power in Lesotho, Cambridge - New-York : Cambridge University Press, 1994.

\section{AUTEURS}

\section{FRANÇOISE DAUCÉ}

CERCEC-EHESS, IUF 